La graduation de droite (M) se rapporte au coefficient relatif aux montants et la graduation de gauche se rapporte aux coefficients relatifs aux croisillons $(\mathrm{C})$.

Le,troisième support caractérise less longueurs de flambage (I) exprimées en centimètres.

\section{Mode D'EMPLOI DU NOMOGRAMME}

Soit à chercher le coefficient de Résal se rapportant à un montant de $200 \mathrm{~cm}$. de longueur de flambage et constitué par une cornière de 6o.6o.6. En joignant le point 200 (support I) au point marqué 6o.6o.6 (Echelle $\mathrm{M}$ ) on trouve sur Je support $\mathrm{S}$ (échelle M) la valeur du coefficient cherché $\mathrm{x}, 34$.

Pour un croisillon de 505.5o.5 (Echelle C, support de gauche) et une longueur de flambage $l=150 \mathrm{~cm}$., nous trourons un coefficient $\mathrm{S}$ (lu sur l'échelle $\mathrm{C}$, support $\mathrm{S}$ ), de 3,55 .

\section{Etablissement du Nomogramme}

La formule que nous nous proposons de traduire graphiquement est

$$
\mathrm{S}=1+\frac{\mathrm{K} l^{2} s}{m \mathrm{I}}
$$

Nous ferons abstraction de la constante I dont nous tiendrons compte au moment de la graduation.

Nous poserons

$$
\mathrm{D}=\frac{\mathrm{K} l^{2} s}{m \mathrm{I}} \text { et } \mathrm{Y}=\frac{k s}{m \mathrm{I}}
$$

$Y$ est constant pour un profil donné et caractérise un profil donné.

L'équation devient :

$$
\mathrm{D}=\mathrm{Y} l^{2}
$$

en prenant les logarithmes:

$$
\log \mathrm{D}=\log \mathrm{Y}+2 \log \mathrm{l}
$$

Construisons les échelles $\log \mathrm{D}$ et $\log l$ avec les mêmes modules. Nous aurons sur le support $l$ une graduation double de cette portée par le support $\mathbf{Y}$.
Pour l'échelle D, nous aurons :

$$
\frac{l}{g}+\frac{l}{g}=\frac{l}{d} \mathrm{~d} \text { 'où } d=\frac{g}{2}
$$

la graduation portée par l'échelle D sera la moitié de celle portée par le support Y.

Le support D sera équidistant de $\mathrm{Y}$ et de $l$.

Graduation. - En graduant et tenant compte du facteur additif $+\mathrm{I}$ - nous obtiendrons la graduation en coefficient de Résal S.

Pour les montants il a suffi d'ajouter + I au chiffre donné par la graduation.

Pour les croisillons il est à remarquer que comme :

$$
\mathrm{I}_{y y} \cdots . \overline{3} \mathrm{I}_{\boldsymbol{x} x}{ }
$$

et que $\mathrm{m}$ (relatif aux montants) $=4 \mathrm{~m}$. (relatifs aux croisillons).

On aura : $\frac{\mathrm{MmI} y y^{\prime}}{\mathrm{Me} \mathrm{I} x x^{3}}=$ ro.

Il suffit donc pour passer de la graduation de gauche $(M)$ à celle droite $(\mathrm{C})$ de retrancher $\mathrm{r}$, multiplier par 10 et ajouter $\mathrm{r}$.

Par exemples : $\mathrm{I}, 3$ devient $(\mathrm{I}, 3-\mathrm{r})$ го $+\mathrm{r}=4$

$$
2,5 \quad(2,5-\mathrm{r}) \quad \mathrm{I} 0+\mathrm{I}=\mathrm{r} 6
$$

Le nomogramme porte en pointillés deux exemples : l'un relatif aux montants, l'autre aux croisillons.

$$
\begin{gathered}
\text { R. VALENSI, } \\
\text { Ingénieur E. S. E. }
\end{gathered}
$$

(1) Voir : Traité de Nomographe, par M. D'OCAGNE (Gauthiervillars, 1899).

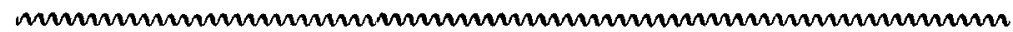

L'abondance des matières nous oblige à renvoyer, à notre numéro Janvier-Février 1921, la fin de l'article de M.Jean EsCard : Imprégnation, Sénilisation el Tgnifugation des Bois d'industrie.

\title{
L'USURE DES TURBINES HYDRAULIQUES
}

\section{SES CONSÉQUENCES ET LES MOYENS D'Y PARER (1)}

L'usure des turbines hydrauliques est un phénomène connu depuis fort longtemps de leurs constructeurs et de leurs propriétaires. Elle a trois causes principales :

ฯ. La construction défectueuse des organes essentiels tels que : distributeurs et roves motrices, tant sous le rapport de leurs dimensions et de leurs formes que sous le rapport de la matière première adoptée ;

2. L'action des éléments chimiques pouvant être contenus dans l'eau ;

3. Enfin, et surtout les alluvions telles que graviers, sables et limons charriés par de très nombreux cours d'eau.

La première de ces trois causes disparait de plus en plus en raison de l'expérience acquise par les constructeurs de turbines dignes de ce nom ; la seconde n’a jamais été très fréquente, mais il faut cependant en tenir compte lors du choix des matières premières entrant dans la construction des turbines, dans les cas où l'analyse de l'eau aurait révélé des éléments nocifs pour le fer et ses dérivés La troisième, par contre, est fréquente, elle le deviendra toujours plus à mesure que la nécessité poussera les pays montagneux, tels que la Suisse, la France et l'Italie (pour

(1) Article paru dans le Bulletin technique de la suisse Romande. ne parler que de ceux nous intéressant de plus près), à utiliser leurs réserves de houille blanche.

M. H. Chenaud, ingénieur, a publié dans les numéros I4, I5, 17, I9 et zo du Bulletin technique de la Suisse romande de l'année rgro, une description très intéressante de l'installation des Forces motrices de la Drance à Martigny, montrant les difficultés surprenantes que peut occasionner aux installations hydrauliques, la grande quantité d'alluvions charriéc par un cours d'eau.

Plus récemment, en $\mathrm{r} 9 \mathrm{i} 6$, M. Ic professeur $D^{r}$ L..W. Collet, alors Directeur du Service des Faux du Département suisse de l'Intérieur, a publié dans le second volume des Annales Suisses d'hydrographie, le résultat de sce études excessivement intéressantes sur le "Charriage des a'luvions dans certains cours d'ean de la Suisse ". Cet ouvrage contient quelques belles photographies d'organes de turbines ruinés nar le sable.

A une époque où les sources d'énergic constituées par nos forces hydrauliques semblent devoir joucr un rôle prépondérant dans le maintien et le progrès du développement génóral de notré pays, i] nous a paru intéressant et utile de présenter aux lecteurs du Bulletin technique les résultats de quelques expériences et travaux consacrés à l'étude des causes et des conséquences de l'usure des turbines hydrauliques, puis aux moyens d'y parer. 
En Suisse, et dans les régions alpines de nos pays voisins, le nombrc de turbines souffrant de l.usure et travaillant avec de mauvais rendements est plus grand qu'on ne le croit. Nous u'hésitons pas à affirmer que la Suisse perd par ce fatt, chaque année, un nombre très respectable de millions de kilowattheures. Cel étal de choses provient, à notre avis, des difficutés rencontrées par les ingénieurs dans l'étude des rendements d'usines hydrauliques en service et du problèmc du dessablage de leur cau motrice

Les circonstances onl voulu que la première usine hydroClectrique, qui à notre connaissance, ait donné lieu à de telles ótudes, fût située sur le flanc ouest de la Cordillère des Andes. Nous présenterons aussi aux lecteurs du Bullelin technique les résultats non moins intéressants de travaux analogues cxécutés récemment dans deux usines de nolre pays. la révision des turbines fit constater leur usure si avancée, qu'il fallut sans retard utiliser les quelques pièces de rechange en éserve. L'examen des bassins de décantation les montra complètement remplis de sable et de limon, l'eau s'ćlant conservée seulement un étroit chenal de chaque côté du grand mur de séparation. Il fallut isoler et vider l'un d'eux au moyen de la vanne de purge prévue dans ce but.; ce travail dura, comme l'on pense, plusieurs jours et il arriva mème une fois que, le débit total devant traverser un seul bassin rempli lui-même d'alluvions, le niveau de l'eau et l'ensablement du canal en amont augmentèrent à tel point que celui-ci déborda.

Les dégâts réparés, on parvint à maintenir le service de l'usine, en nettoyant alternativement et sans intervalle aueun, les deux bassins, mais il devint évident que les moyens dont on disposait étaient insuffisants pour la lutte contre la quantité formidable

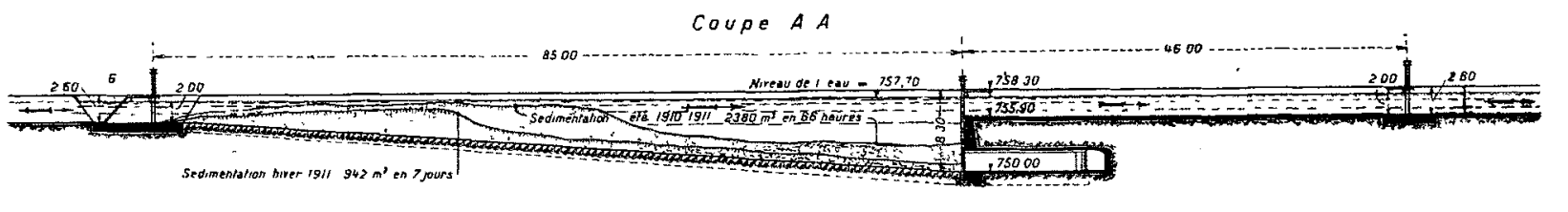

Coupe $B B$

Coupe $C$ C.

Coupe $D O$.

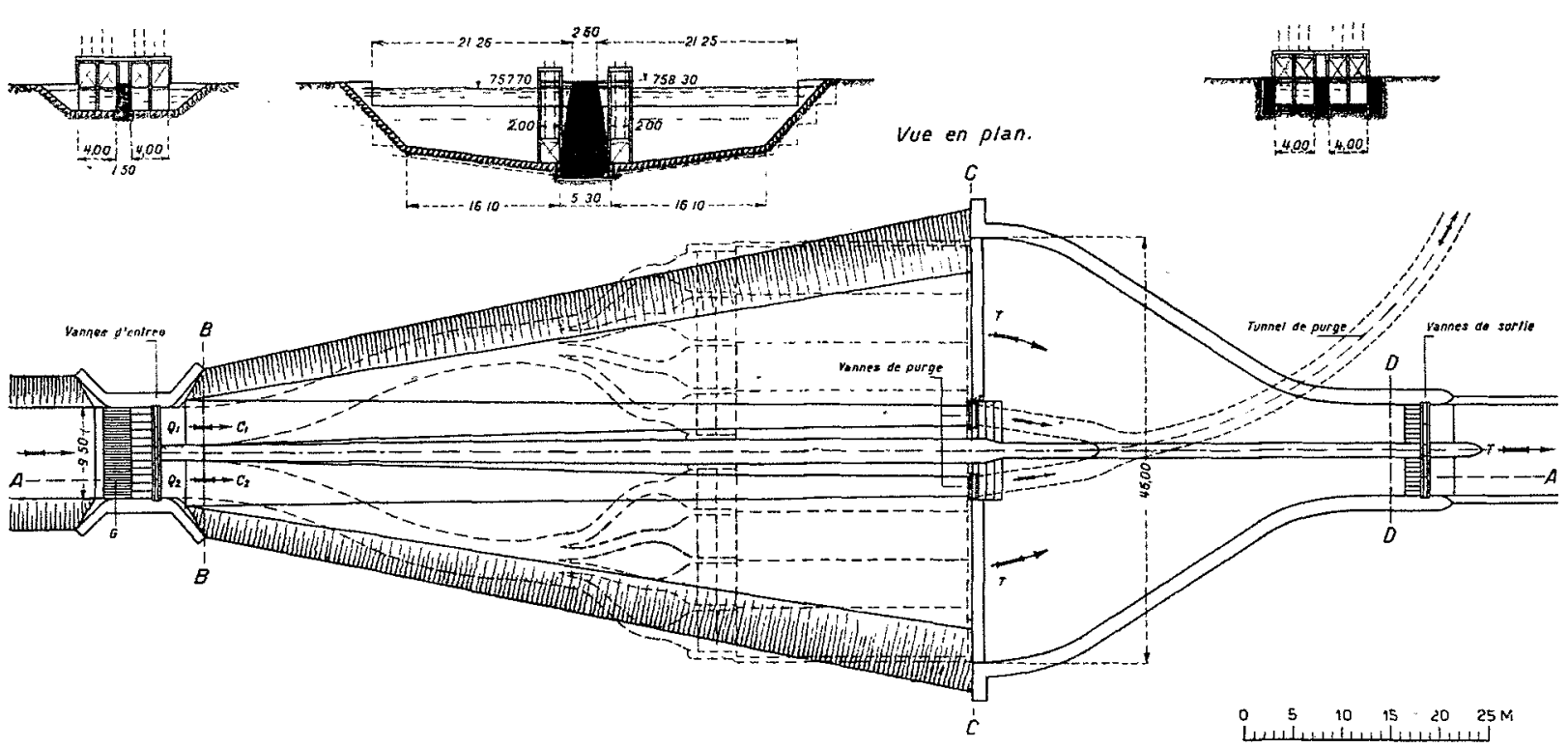

Pig. 1. - Bassins prmmis de décantalion de Florida-Alta.

\section{I. - Etudes e't travaux a l'Usine mydro-Électrioue} de Santiago du Chili a Florida-Alta.

Cette installation destinée à alimenter la capitale du pays en énergie électrique, est située à $18 \mathrm{~km}$. au sud de la ville ; elle est artionnée par l'eau du grand canal d'irrigation "San Carlos " dont le débit dépassant à certaines époques $60 \mathrm{~m}^{3}$ par seconde, est dérivé du "Rio Maipo ". Il est intéressant de noter ici que l'usine doit restituer au canal San Carlos toute l'eau détournée et que ce débit de $60 \mathrm{~m}^{3}$ par seconde, très respectable pour un canal d'irrigation, est utilisé avec une parcimonie des plus frappantes, jusqu'au dernicr litre. pour fertiliser le pays

Construite dans les années rgoz à rg10 par la" "Compania Alemana Transatlantica de "Electricidad à Santiago " (DeutschUeberseeische Elektriżitäts-Gesellschaft in Berlin), l'usine dispose d'un débit de $20 \mathrm{~m}^{3}$ par seconde sous une chute brute de $98 \mathrm{~m}$. ; elle est prévue pour six groupes de turbines avec alternateurs triphasés de 4.00o HP, dont un de réserve et possédait, dès le débul, pour le dessablage de son eau, deux grands hassins de décantation représentés par la fig. i De la vanne d'entrée à la vanne de purge, chacun do ces bassins a une longueur de $85 \mathrm{~m}$., une largeur maximum de $33,5 \mathrm{~m}$. el une profondeur de $8,3 \mathrm{~m}$. dimensions qul, même pour un débil de ro $\mathrm{m}^{3}$ par sceonde et par bassin, peuvent paraître trìs suffisantes

I a première période de service : mai-octobre 1910 , tombant pour l'hémisphère sud sur les mois d'hiver, ne révéla aucun inconvénient grave des installations, mais en janvier et février rgr, d'alluvions amenées par l'eau et détruisant à brève échéance les quatre turbines alors installées.

La fig. 2 montre l'aspect d'une partie du bassin de droite pendant le lavage dù dépôt d'alluvions, atteignant plusieurs mètres de hauteur. L'eau s'échappant par la vanne d'entrée entraîne peu à peu le pied du dépôt vers la vanne de purge et de là par un canal spécial, rejoignant le trop-plein du château d'eau, au canal San-Carlos.

Les turbines avaient été construites tout spécialement pour les conditions difficiles de cette usine par les deux plus grandes et meilleures fabriques de turbines europécnnes

Sur la demande de la Société exploitant l'usine - La "Chilian Electric Tramway and Light. $\mathrm{C}^{\circ}$ Limited " à Santiago, les deux constructeurs de turbines furcnt invités à envoyer un de leurs ingénieurs pour étudier sur place cette grave question et tout spérialement une modification éventuelle des types de turbines adoptés.

Délégué par la maison bien connue : J.-M. Woith, à Hcidenheim, qui avait fourni les turbines $N^{\text {os }}$ III et IV, avec la mission de faire re qui était en notre pouvoir pour obvier aux incónvénients signalés. nous dûmes bientôt constater que Jes turbines s'usaient uniquement sous. l'action mécanique du sable contenu dans l'eau. Les pièces soumises à l'usure, telles quc les distributeurs et les roues motrices; étant en acier, voire même en acier au nickel et en bronze spécial, ne pouvaient ĉtre construites d'un type différent ou en matières plus résistantes; il fallait donc 
chercher à supprimer la cause de cette usure, c'est-à-dire le moyen de dessabler l'eau motrice. Cette opinion était également cellc du personnel dirigeant des doux sociétés nommées plus haut.

Les résultats de nos premières constatations furent très intéressants. Dans la coupe $A-A$, fig. I, d'un bassin de décantation est indiqué le profil d'un volume d'alluvions de $942 \mathrm{~m}^{3}$ déposé en sept jours de l'hiver ig̣r. Le volume d'ean ayant passé par le bassin étant de $6 \mathrm{~m}^{3} \mathrm{par}$ seconde, la quantité d'alluvions déposée avait été en moyenne de :

$$
\frac{942 \cdot 1000 \cdot 1000}{6.1000 \cdot 7 \cdot 24 \cdot 3600}=0,26 \mathrm{~cm}^{3}(=\text { environ } 0,39 \mathrm{gr} .)
$$

par litre d'eau.

Dans la môme figure est indiqué le profil d'un volume d'alluvions de $2380 \mathrm{~m}^{3}$ déposé en 66 hcures de l'été IgIo-Igri. Le volume d'eau ayant passé par le bassin étant de $5,08 \mathrm{~m}^{3}$ par scconde, la quantité d'alluvions déposée avalt été en movenne de :

$$
\frac{2380 \cdot 1000 \cdot 1000}{5,08 \cdot 1000 \cdot 66 \cdot 3600}=2 \mathrm{~cm}^{3}=\text { (environ } 3 \mathrm{gr} \text {.) }
$$

par litre d'eau.

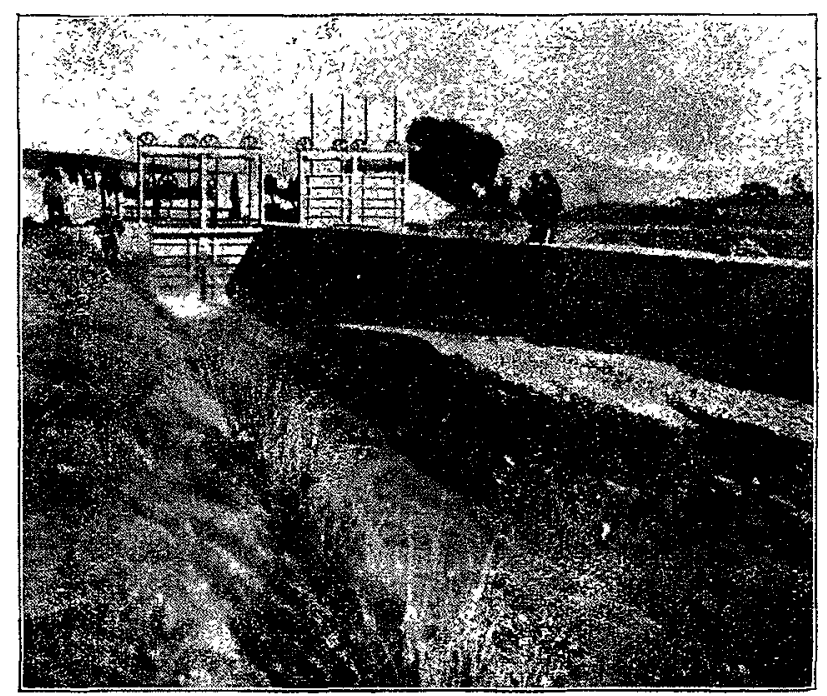

Fug. 2. - Aspect d'un bassin pendant le lavage du dépôt d'alluvons

Comme le lecteur le comprendra, ces deux chiffres ne donnent que la quantité moyenne d'alluvions déposée par l'eau lors de son passage au travers des bassins de décantation. Ils ne sont ni des minimums ni des maximums, mais peuvent cependant servir de base pour se faire une idée approximative de la quantité d'alluvions traversant journellement les turbines lorsque les bassins de décantation n’étaient pas vidés au moment voulu.

Voici ces quantités :

Pendant l'hiver rgrt, le débit moyen de l'usine ayant été d'environ $\mathrm{x}_{2} \mathrm{~m}^{3}$ par seconde, la quantité d'alluvions ayant traversé les turbines aura donc dépassé certains jours :

$$
-\frac{0,26 \cdot 1000 \cdot \mathrm{x} 2 \cdot 3600 \cdot 24}{1000 \cdot 1000}=260 \mathrm{~m}^{3}
$$

Pendant l'été où le débit de l'usine allait être, par la suite de эo $\mathrm{m}^{3}$ par seconde, la quantité d'alluvions qui allait traverser les turbines dépasserait :

$$
\frac{2 \cdot 1000 \cdot 30 \cdot 3600 \cdot 24}{1000 \cdot 1000}=3456 \mathrm{~m}^{3} \text { par jour. }
$$

Nous nous permettrons de remarquer ici que le volume d'alluvions déposé dans les bassins : $2 \mathrm{~cm}^{3}$ par litre d'eau, donnant pour le débit de $20 \mathrm{~m}^{3}$ par seconde un volume énorme de $3 / 56 \mathrm{~m}^{3}$ par jour, n'a rien d'excessif et est inférieur aux chiffres que l'on obticndrait en Suisse. D'après la publication de $M$. Je professeur I..W. Collet, citée plus haut, le volume des alluvions cn suspension dans l'eau de la Drance a Martigny-Bourg fut, pour les mois de juillet et août 1909 , en moyenne de $4,07 \mathrm{~cm}^{3}$ avec un maximum journalier d'environ $22 \mathrm{~cm}^{8}$ par litre. L'eau de lá
Borgne à Bramois est encore plus chargée puisqu'elle contenait, en juillel et août 1909 , en moyenne $5,6 \mathrm{~cm}^{3}$ avec un maximum d'environ $23,4 . \mathrm{cm}^{3}$ par litre. Le 17 juin $\mathrm{rg} 8 \mathrm{8}$, jour de pluie, nous avons trouvé dans l'eau de la Saltine à Brigue $4,2 \mathrm{~cm}^{3}$ par litre.

Ces chiffres ont une très grande importance pratique, car ils permettent de calculer ì l'avance la quantité d'alluvions qui passeront par les turbines d'une usine que l'on veut construire. Si l'on prend par exemple une teneur en alluvions de $4 \mathrm{~cm}^{3}$ par litre et un débit de $5 \mathrm{~m}^{3}$ par seconde, on obtient pour 34 heures :

$$
\frac{4 \cdot 5 \cdot 1000 \cdot 3600 \cdot 24}{1000 \cdot 1000}=1728 \mathrm{~m}^{3}
$$

ou :

$$
\frac{1728 \cdot x, 5 \cdot 1000}{10 \cdot 100}=259 \text { vagons de ro tonnes. }
$$

On est en droit de s'étonner que les turbines puissent résister pendant plusieurs mois d'été à l'action destructive de pareilles masses d'alluvions. Ces chuffres montrent aussi, nous semble-t-il, l'impossibilité de lutter efficacement contre les aliuvions avec des bassins ou chambres de décantation ordinaires sans écoulement automatique des alluvions éliminées.

Les alluvions trouvées dans les bassins de Florida-Alta consistaient en sable dont le diamètre des plus gros grains ne dépassait guère $5 \mathrm{~mm}$, en limon et en une boue de couleur rougeâtre. Seuls, quelques fragments isolés de lave rolcanique attcignaient so et 20 millimètres de diamètre.

Ces constatations permettaient de conclure sans aucun doute, que l'usure des turbines était un phénomène absolument naturel et indépendant de leur type et de-leur qualité.

L'examen plus approfondi des alluvions trouvées dans l'eau uous fil volr que les éléments dont la vitesse de précipitation, dans un tube rempli d'eau, était inférieure à $30 \mathrm{~mm}$. par seconde, vitesse correspondant à un diamètre des grains allant jusqu'à o,4 $\mathrm{mm}$. paraissaient, vu leurs petites dimensions et leur nature en partie plus tendre, être pcu dangereuses pour les turbines.

Le problème qui se posait était donc : trouver un dispositif capable d'éliminer de l'eau motrice, tous les troubles dont le diamètre des grains- dépassait $0,4 \mathrm{~mm}$. et permettant l'évacuation rapide et peu conteuse des quantités considérables de sables et limons auxquelles il fallait s'attendre.

Pour provoquer la précipitation des alluvions à éliminer, nous cûmes l'idée d'utiliser le dispositif imaginé et exécuté par M. A. Boucher, ingénieur à Prilly, au dessableur de l'usine des forces motrices de la Drance à Martigny. Ce dispositif destiné à donner à l'eau une vitesse ascendante très faible, nous parut susceptible d'un perfectionnement, consistant à donner aux bas= sins de décantation d'autres proportions, puis à exécuter les parties supérieures et inférieures des paroịs-guides transversales, déplaçables verticalement, ceci pour pouvoir en régler la hauteur et obtenir une distribution ef une vifesse ascendante de l'eau; uniforme sur toute la surface des bassins.

Pour faciliter l'évacuation des alluvions qui se précipiteraient vers le fond et en obtenir si possible l'écoulement antomatique ct continu, nous nous proposions de donner au fond des bassins une section transversale en forme d'entonnoir et de munir sa partic la plus profonde d'un certain nombre d'orifices purgeurs qui laisseraient échapper les alluvions avec une certaine quantité d'eau.

Le dispositif réalisant ces idées fut construil sous la forme d'un dessableur d'essai représenté par les fig. 3 et 4 . La figure 3 est une reproduction du dessin d'exécution, la fig. 4 une vue du dessableur en activité. Les dimensions de la parlie du bassin dans laquelle l'eau se clarifie pendant son mouvement ascendant étaient - longueur $4 \mathrm{~m}$., largeur $\mathrm{T} \mathrm{m}$. 25 , profondeur $2 \mathrm{~m} .40$. Les volumes d'eau devant être, à l'arrivée : eau à dessabler, I 68 litres/seconde ; au départ : eau dessabléc, r5o litres/seconde ; eau de purge, $168-{ }_{1} 50=18$ litres/seconde, la vitesse moyenne de l'ean montant dans le bassin scrait de:

$$
\frac{0,150}{4,0 \cdot 1,25}=0,03 \mathrm{~m} . \text { par seconde. }
$$


Après quelques tâtonnements dans l'exécution correcte de ses détails intérieurs, les résultats de cette première application furent excellents. Il était visible que tout le sable contenu dans l'eau à l'arrivée se précipitait et s'écoulait par les orifices pur- l'expérience $\mathrm{N}^{\circ} \mathrm{ro}$, il y a lieu de remarquer que les alluvions contenues dans l'ean consistaient essentiellement en limon fin et léger. A l'exception de quelques dépôts, se formant et se stabilisant dans les angles morts du fond et des côtés, la totalité des
Coupe A-A

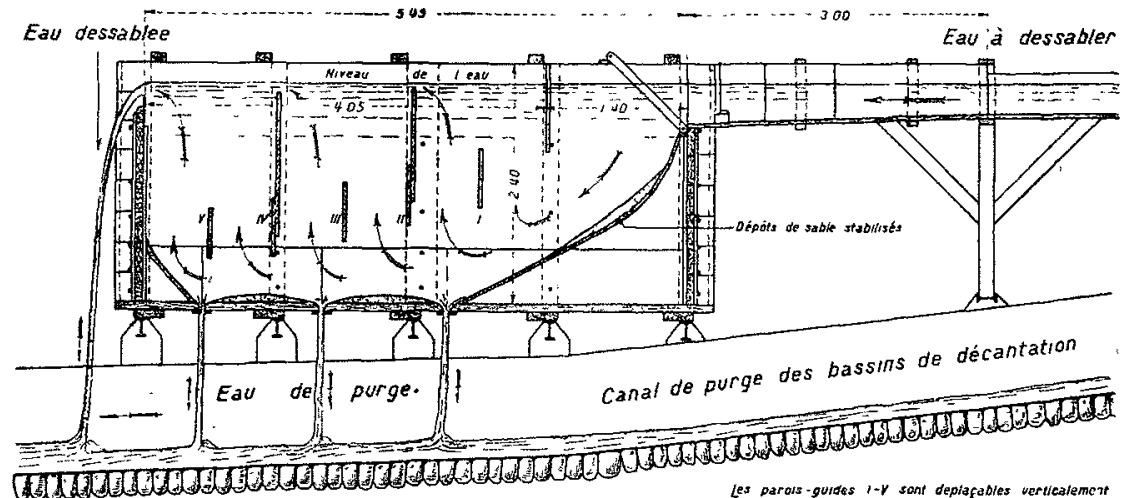

Vue en plan.

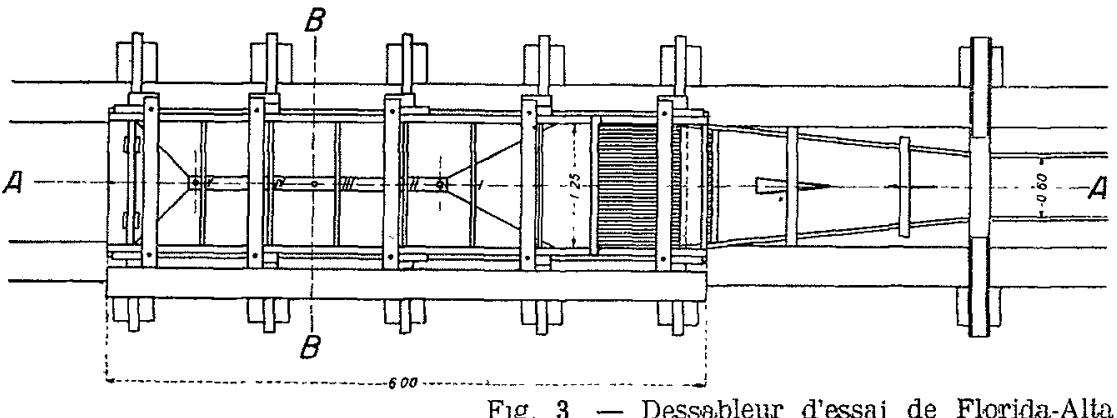

Coupe $B-B$

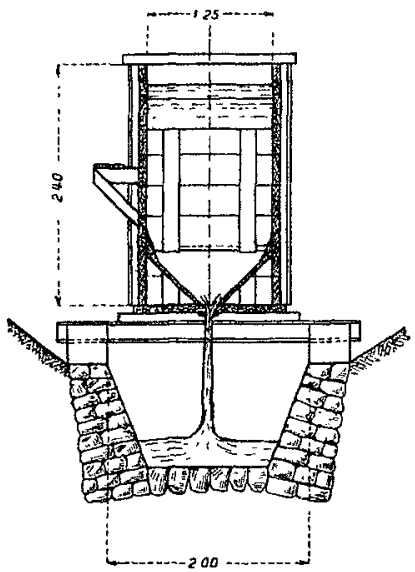

geurs du fond. La mise au point des parties supérieures et inférieures des parois-guides transversales par leur déplacement verlical, permit d'obtenir une vitesse ascendante de l'eau bien uniformément répartie, condition essentielle pour réalisè la précipitation des troubles jusqu'à la limite que nous nous étions fixée.

Les expériences de dessablage exécutées en présence des ingénieurs des sociétés intéressées furent très probantes ; en voici les résultats :

\begin{tabular}{|c|c|c|}
\hline Numéro de l'expérience & $\begin{array}{c}\text { Teneur en alluvions } \\
\text { de l'eau à dessabler } \\
\mathrm{cm}^{2} \text { par litre }\end{array}$ & $\begin{array}{c}\text { Teneur en alluvions } \\
\text { de l'eau dessablée } \\
\mathrm{cm}^{3} \text { par litre }\end{array}$ \\
\hline $\mathrm{I}$ & 0,8 & 0,02 \\
2 & 0,9 & 0,07 \\
3 & $\mathrm{I}, \mathrm{o}$ & 0,17 \\
4 & $\mathrm{r}, 5$ & 0,05 \\
5 & $2, \mathrm{I}$ & 0,32 \\
6 & 3,6 & 0,32 \\
7 & 33,0 & $x, 40$ \\
8 & 39,0 & 0,92 \\
9 & 39,0 & $x, 90$ \\
$\mathrm{I}$ & 67,0 & 5,80 \\
\hline
\end{tabular}

C'étail un plaisir de yoir s'écouler le sable par les orifices purgeurs. Lors des dernières expériences, par exemple, l'eau sortant de l'orifice voisin de l'entrée contenait par moment $60 \%$ de sable.

L'examen des alluvions restant dans l'eau dessablée montra que la vitesse de précipitation des grains les plus gros ne dépassait pas $35 \mathrm{~mm}$. par seconde et leur diamètre environ o,5 mm., d'où il résultait que le degré de dessablage obtenu pratiquement était peu inférieur à celui théoriquement possible. Au sujet de alluvions éliminées par le dessableur fut évacuée automatiquement et de façon continue.

Ces résultats montraient que la solution du problème était trouvée et qu'il ne restait plus qu'à utiliser les expériences faitès

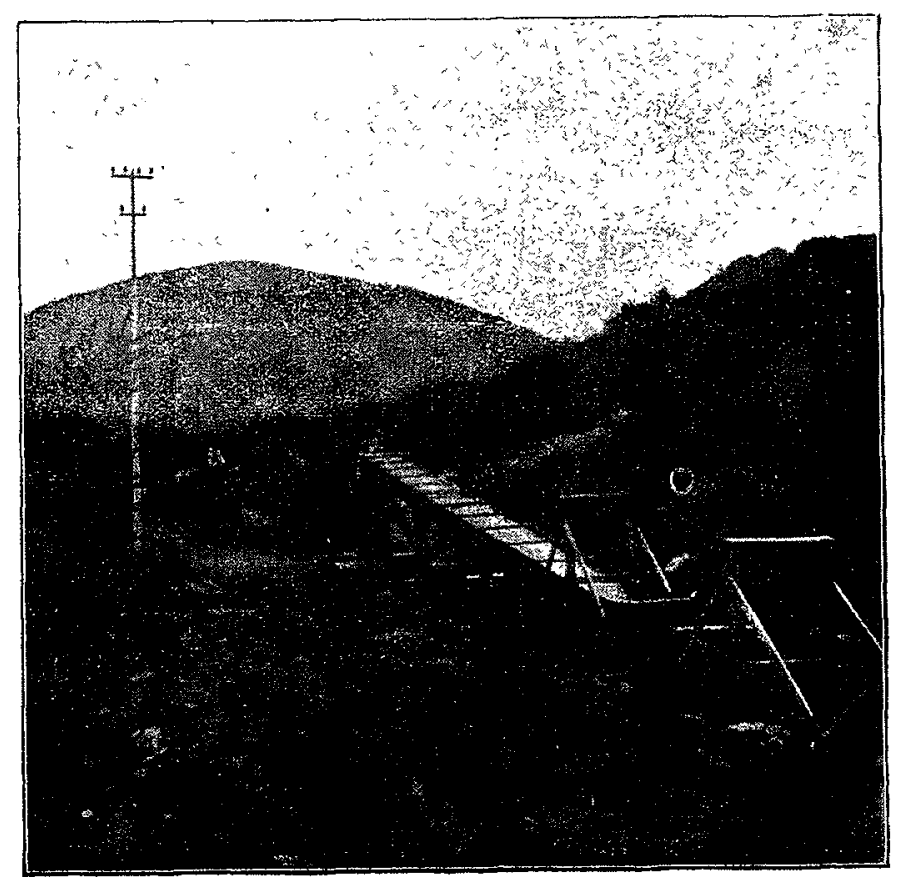

Fig. 4. - Vue du dessableur d'essar et de son canal d'amenée en activité

cn les reportant à une installation de dimensions plus grandes. Nous fûmes chargés d'élaborer le projet pour deux dessableurs de service devant être placés dans les excavations des bassins de décantation existants et dessabler chacun un débit de $10 \mathrm{~m}^{3}$ par seconde. 
Les expérierices faites entre temps sur le canal d'amenée avaient démontré que sa pente était suffisante et qu'avec un dessableur du nouveau lype l'ensablement ne se produrait plus.

Les aväntages de ce nouveau type de dessableur allaient donc ctre : de supprimer l'ensablement du canal d'amenée, d'augmen. ter de façon intense lc dessablage de l'eau et de réduire considérablement les frais de cette opération puisque la manutention des dessableurs ne comporteralt plus que la survellance générale ct to nelloyage de la grille. Pour les turbines, on pouvail s'allendre à une longévité plus grande des pièces soumises à l'usure et à une plus grande sécurité du-service, avantages très précieux dans un pays comme le Chıli où les pièces de réserve doivent ètre tirées d'Europe et où le personnel capable de réparer des turbines est difficile à obtenir.

Ces avantages fort plausıbıes en eux-mèmes étaient cependant difficiles à exprimer par des chiffres qui eussent pu justifier de façon toute objective la dépense et le risque que comportait la construction des deux grands dessableurs d'un système perfectionné, apparemmeni compliqué et complètement nouveau.

(4 suivie.) Henri Dufour, Ingénieur.

\section{L'UTILISATION DES COMBUSTIBLES}

\section{Comment les usines à gaz peuvent parer à la pénurie du charbon (1).}

A la sulte des difficultés de transport, un grand nombre d'usines à gaz ont dû arrêter leur production ces derniers temps. Par: mi celles-ci, signalons celles de Carpentras, Tourmon, Bagnols, Paray-le-Monial, Cours, etc... Comme il semble bien que cetlo pénurie doive ètre, pendanl quelques années encore, considérée comme un étal endémique, il semble prudent pour les usines a gaz de s'outiller pour y parer dans la mesure du possıble. Nous voudrions indiquer aujourd'hui un procédé permetlant d'augmenter considérablement le readement du charbon en gaz sans modilications bien considérables. Il consiste essentıellement à empêcher la décomposition des hydrocarbures qui habituellement sćjournent dans la cornue un temps trop considérable au contact des parois portées à haute température. Le résultat immédiat est un dépòt compact de charbon dit de cornue qui, tout en diminuant la richesse du gaz produit, forme un enduit isolant, augmente l'épaisseur de la cornue et oblige à employer, pour le chauflage, une quantité excessive de coke.

Ce balayage s'opère au moyen d'un mince filet de vapeur surchauffée introduit, s'il s'agit de cornues horizontales, par l'arrière de celles-ci. Cette vapeur, au contact du charbon, produit du gaz à l'eaì lequel, par son volume, dilue les hydrocarbures et les entraîne rapidement hors de la cornue. Des essais que nous avons pratiqués, il résulte que le réndement en gaz passe de 30o-35o mètres cubes à 550-650 mètres cubes à la tónne, sans que pour cela le pouvoir calorifique du gaz soit abaissé dans des proportions excessives. Il conserve au mètre cube de 4.200 à 4.400 calories au lieu de 4.800 que donne la distillation, habituellement pratiquée avec les charbons dont on peut disposer actuéllement. On parvient, ainsi à extraire d'une tonne de houille environ 2.500 .000 calories sous forme de gaz au lieu de 1.250 .000 calories qui constituent un -rendement normal.

On s'explique 'la richesse du' gaz ainsi obtenu par le róle protecteur que joue le gaz à l'eau vis-à-vis des carbures benzéniques et méthaniques. Ceux-ci, habituellement se décomposent sous l'influence d'une haute température, en-donnant naissance au charbon de cornue, au gràphite, à la naphtaline, à l'anthracène et autres carbures à poids moléculaires élevés dont on connaît l'influence désastreuse dans l'entretien des tuyauteries où ils se déposent. Ce sont les carbures légers qui enrichissent suffisamment le gaz pour lui permettre un pouvoir calorique encore élevé On observe d'autre part la formation de goudrons beaucoup plus fluides et la diminution de la proportion de brai. On peut comp-

(1) Extrait de la Revue industrielle de l'Est. ter sur ume moyeune de 5 i $6 \%$ de goudron. Cette quantité, dans un four à sept cornues bren dirigé. permet son chauffage à peu prìs complet par l'emplor d un bon brúleur à goudron, fonctionnanl avec un très faible excès d'uir. On an, constale jamais aucun encrassement des cornues, mass il ęst certan que l'on perd, par suite de la porosité de cclles-cl, une petıte quantite d'hydrogène.

br, a la sulte du lour, on a som de piaces unc recuperation d'air chaud ansi quine petite chaudice, 11 est pussible de produne toute la vapeur necessane au lonchonnement de l'usme a gat el ae ne labser sortir les lumees qu a jo0" au lieu de 1,000 is

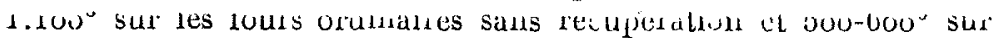

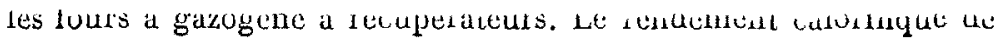

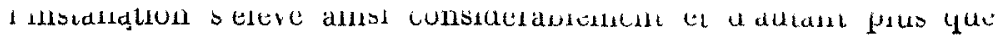
lon prend, dans ld constiuction du luar, ues precaluons a 150 lement caloriaque ventabenent trop hughoes habltatiement.

un autce arallage au procede est a upe. el automaliquemenc lo netroyage du coke de sa poussiere. 1 res liequimment, arej les noulles deplorables que 1 on dut distiller peildant la guere, il arrivait que te coke onlenu étall très poussiereux. Il l'elail d'autaut plus que lon completant les charge de con nues avec de la scrure et des debris de bols. Ur, l'emploi d une injecion contirue de vapeur permet la destruction de tout ce poussicr qui se trouve le premier altaqué. un peut done, a la rigueur, oblenrr un mème rendement en coke quautrelors avec ce procédé si l'on a soin de mélanger la houlle d'une quantité de sclure sufinsante pour que le chartuon de boss formé pulsse décomposer la quanth de vapenr d'eau introdute.

A notre connassance, ce procédé trop peu commu en iriunce, 1: a été employé sur cornues horizontales que dans une seule usine. Il est en montage dans une seconde usine. Par contre, en Angleterre, on laurait depuss peu appliqué à de nombreuses connues verticales et les résullats qu'il donne amsı sont absolument identiques à ceux que nous avons obtenus nous-memes.

Ajoutons que l'application de ce procedé demande beaucoup de soins, pour éviter de trop refrondır les fonds de cornue ce qui pourrait entraîner leur crevassemcnt. Bien appliqué, il permet des économies de charbon très élevées et fournil la même quantité de coke que la distillation sans vapeur, étant donné l'énorme augmentation de production de chaque four el la réduction concomittante du coke employé pour le chaulitage. Enfin, par l'emploi du brûleur à goudron, on simplitie singulièrement la surveillance du chaulfage des fours toujours délncate de nuit et l'on peut réduire la mam-d'œuvre à sa plus simple expression.

Dans le cas du chauffage au coke, on peut améliorer considérablement le rendement par l'emploi de foyers divergents formant une chambre de combustion plus vaste avec voûte de grand sayon. On peut également, très simplement, obtenir le réchauffage de l'air einployé a la combustion. On fait pénétrer celui-ci par l'arrière du cendrier, ce qui permet do lui faire parcourir une galerie étroite à chrcanes où il s'échauffe all contact des fumées quittant le four par le carneau se rendant à la chcminée. Les foyers devieunent blancs et les grilles très épaisses se ramollissent et se déforment très vite si l'on n'a soin d'alimenter le cendrier en eau Celui-ci cst, bien enterdu, hermétiquoment clos a l'avant.

Il semble que dans la généralité des cas, avoc une diminution concomiltante du prix du mire cube, les villes accepteraient ce mode de fabrication Etant donné même la pénuric du charbon en France, nous estimons que l'emploi de ce procédé s'impose, parce que plus économique que tont antre, il peut ètre installé sans grandes modifications sur tous les genres de fours.

Ajoutons qu'il permet l'emploi de houjlles très médiocres at même de charbons barrés. Toulefois le pouvoir calorifique du gaz obtenu s'abaisse scnsiblement avec la qualité du combustible.

H. Contaut.

\section{La récupération de l'énergie sur les chemins de fér} electriques à la descente des pentes $\left(^{1}\right)$.

Dans les circonstances actuelles, où les économies en combus. tible s'imposent d'une façon sí intense, la question de la récupé-

(1) Extrait de la Revue indusirielle de l'Esl. 